\title{
Radiative characterization of random fibrous media with long cylindrical fibers: Comparison of single- and multi-RTE approaches
}

\author{
Jaona Randrianalisoa ${ }^{\mathrm{a}, 1, *}$, Sophia Haussener ${ }^{\mathrm{b}, 1, *}$, Dominique Baillis ${ }^{\mathrm{c}}$, Wojciech Lipiński ${ }^{\mathrm{d}}$ \\ a Groupe de Recherche en Sciences pour l'Ingénieur (GRESPI), EA 4694, Université de Reims Champagne-Ardenne, Campus du Moulin de la Housse, F-51687, \\ Reims, France \\ ${ }^{\mathrm{b}}$ Institute of Mechanical Engineering, Ecole Polytechnique Fédérale de Lausanne, CH-1015, Lausanne, Switzerland \\ ' Laboratoire de Mecanique des Contacts et des Structures (LaMCoS), UMR CNRS 5259, INSA de Lyon, 18-20 Rue des Sciences, F-69621, Villeurbanne Cedex, \\ France \\ d Research School of Engineering, The Australian National University, Canberra, ACT 2601, Australia
}

\section{A R T I C L E I N F O}

\section{Article history:}

Received 16 March 2017

Revised 17 July 2017

Accepted 1 August 2017

Available online 3 August 2017

\begin{abstract}
A B S T R A C T
Radiative heat transfer is analyzed in participating media consisting of long cylindrical fibers with a diameter in the limit of geometrical optics. The absorption and scattering coefficients and the scattering phase function of the medium are determined based on the discrete-level medium geometry and optical properties of individual fibers. The fibers are assumed to be randomly oriented and positioned inside the medium. Two approaches are employed: a volume-averaged two-intensity approach referred to as multiRTE approach and a homogenized single-intensity approach referred to as the single-RTE approach. Both approaches require effective properties, determined using direct Monte Carlo ray tracing techniques. The macroscopic radiative transfer equations (for single intensity or two volume-averaged intensities) with the corresponding effective properties are solved using Monte Carlo techniques and allow for the determination of the radiative flux distribution as well as overall transmittance and reflectance of the medium. The results are compared against predictions by the direct Monte Carlo simulation on the exact morphology. The effects of fiber volume fraction and optical properties on the effective radiative properties and the overall slab radiative characteristics are investigated. The single-RTE approach gives accurate predictions for high porosity fibrous media (porosity about 95\%). The multi-RTE approach is recommended for isotropic fibrous media with porosity in the range of $79-95 \%$.
\end{abstract}

(c) 2017 Elsevier Ltd. All rights reserved.

\section{Introduction}

The most common approach to solve radiative transfer problems in heterogeneous, morphologically complex materials is to solve the Radiative Transfer Equation (RTE) by treating a disperse medium as one continuous and homogeneous medium having apparent radiative properties [1,2]. The determination of the apparent radiative properties of dispersed media based on media morphology and composition becomes crucial to the accuracy of this approach. This topic has continuously attracted interest from the radiative transfer community due to its large variety of applications such as solar energy conversion [3-5], nuclear technologies [6], snow physics [7], and medical applications [8]. The reviews of Viskanta and Mengüç [9], Baillis and Sacadura [10], and Bail-

\footnotetext{
* Corresponding author.

E-mail addresses: jaona.randrianalisoa@univ-reims.fr (J. Randrianalisoa), sophia.haussener@epfl.ch (S. Haussener).

1 Equally contributing.
}

lis et al. [11] present the theoretical approaches and experimental methodologies for the characterization of spectral, directional apparent radiative properties of particulate and realistic disperse media, such as foams, fibrous materials, various ceramics, polymer coatings containing microspheres, and aerogel super-insulations. This traditional approach becomes less accurate if the dispersed medium has larger volume fractions, is percolating, and exhibits semitransparent instead of opaque behavior. Different intensities for each component and phase are expected and have to be considered. A theoretical multi-RTE approach, i.e. averaged radiative transfer equations are valid in each individual phase, has recently been introduced within the limit of geometrical optics assumptions [12-18]. This approach has the ability to incorporate realistic and morphologically complex media. As an example, this approach was used by Haussener et al. [7] for the determination of the macroscopic properties of different types of snow, a dispersed ice medium semitransparent in the visible wavelength range. In this previous study, a direct comparison between multi-RTE approaches and single-RTE approaches had been conducted, indicating that 


\section{Nomenclature}

c fiber clearance

d fiber diameter

$f_{\mathrm{s}} \quad$ boundary fiber surface fraction

$f_{\mathrm{V}} \quad$ fiber volume fraction

$g \quad$ asymmetry factor of scattering

$I_{i} \quad$ volume-averaged intensity

$k_{i} \quad$ imaginary part of complex refractive index

$L \quad$ intensity in Eq. (1); edge length of fibrous samples

$m \quad$ complex refractive index

$n \quad$ real part of complex refractive index

$N$ number of Monte Carlo rays

p position on the fiber axis

$P_{\text {sca }} \quad$ probability of scattering

$P_{\text {sca }}(\Theta)$ angular probability of scattering

$q \quad$ radiative flux

$q_{\text {in }} \quad$ incident radiative flux

$Q_{\text {ext }} \quad$ extinction efficiency factor of a single fiber

$R \quad$ reflectance

$s \quad$ path length in Eq. (8)

$\hat{\boldsymbol{s}} \quad$ radiation direction unit vector

$T$ temperature

$\hat{\mathbf{t}} \quad$ direction unit vector of the fiber axis

Tr transmittance

$V \quad$ representative elementary volume

$x \quad$ fiber size parameter

$\mathbf{x}$ position vector of the intensity

$x, y, z \quad$ Cartesian coordinates

\section{Greek symbols}

$\beta \quad$ extinction coefficient

$\Phi \quad$ scattering phase function

$\kappa \quad$ absorption coefficient

$\lambda$ radiation wavelength

$\Lambda \quad$ extinction mean free path

$\rho \quad$ reflectivity

$\sigma \quad$ scattering coefficient

$\Theta \quad$ scattering angle

$\omega \quad$ scattering albedo

$\Omega \quad$ solid angle

\section{Superscripts}

$+\quad$ forward directions

- backward directions

Subscripts

a effective radiative properties of the single-RTE approach

b blackbody

$i \quad$ index of medium component (1 - host, 2 - fibers)

in incoming intensity

ind independent scattering

int intrinsic

refl reflexion

$\lambda \quad$ wavelength

the accuracy of the simplified one-phase approach strongly depends on the sample morphology, i.e. characteristics sizes, volume fractions, and properties.

Although fibrous materials have been widely investigated as lightweight thermal insulators [19-25], they are interesting materials for other applications such as combustion support in burners [26], solar fuel production [27], and thermal protection systems $[28,29]$ among others. Earlier investigations of radiative transfer in fibrous media focused mainly on infinitely long fibers with sizes comparable or smaller than the radiation wavelengths (e.g. [1925]) while few studies focused on radiative characteristics of optically large fibers [29-33]. Recent works of Dauvois et al. [33] focused on the implementation of the multiple intensities approach in order to study radiative transfer in a felt of fibers applied as insulation in high-temperature systems. They discussed the suitability of the Beerian approach and the radiative Fourier's model for semitransparent fibers in a transparent medium. Our contribution follows the study presented in [33].

In this study, we aim at comparing traditional radiative transfer approach based on single intensity, widely detailed in standard textbooks (e.g. [1,2]), to multiple intensities approach, previously applied to packed beds (e.g. [6,34-36]), foams (e.g. [14,18]) and fibrous media, [33] in order to estimate the applicability of simplifying theoretical approaches for predicting radiative transfer in isotropic fibrous materials consisting of optically large fibers. Two approaches are employed: (1) a volume-averaging approach for the calculation of averaged intensities in the multiple phases, referred to as multi-RTE approach, and (2) a single intensity approach, referred to as single-RTE approach. The first approach enables computing effective properties for each component of the multi-phase system by utilizing the probability distribution function. The second approach provides effective properties of the equivalent homogeneous system. The latter properties are determined by applying the mean-free-path theory. The effective radiative properties obtained for two approaches are then used to compute the overall transmittance and reflectance of the medium and radiative flux distribution by solving the corresponding (single or volume averaged two) RTEs. The results are compared against predictions by the direct simulation Monte Carlo (DSMC) (e.g. as presented in [32]).

\section{Problem statement}

Radiative characteristics are determined for fibrous media consisting of randomly positioned and oriented long cylindrical fibers. The model medium is schematically shown in Fig. 1(a). It consists of circular cylinders representing the fibers, comprised in a cubic control volume $V$ of edge length $L$. The edge length $L$ is taken sufficiently longer than the fiber diameter $(L / d>>10)$ to minimize boundary effects. A single fiber is defined by the direction of its axis, $\hat{\mathbf{t}}$, a point on the axis, p, and the fiber diameter $d$ (Fig. 1(b)). The fibers have a volume fraction $f_{\mathrm{v}}=V_{\mathrm{f}, \mathrm{t}} / V$ within the control volume with $V_{\mathrm{f}, \mathrm{t}}$ being the total volume of the fibers.

Three-dimensional geometries of the model fibrous medium of selected $d$ and $f_{\mathrm{v}}$ are constructed by generating each fiber by choosing randomly $\mathbf{p}$ in the control volume $V$ and $\hat{\mathbf{t}}$. If a newly generated fiber overlaps with the existing ones, it is discarded and another fiber is generated. Fiber volume fraction up to $21 \%$ are considered in order to avoid difficulties associated with fiber overlap during the geometry generation.

We consider samples with three different porosities namely $95 \%$ $\left(f_{\mathrm{v}}=0.05\right), 85 \%\left(f_{\mathrm{v}}=0.15\right)$, and $79 \%\left(f_{\mathrm{v}}=0.21\right)$. To account for the finite sample size and the corresponding variations in the morphological properties in the medium, we generate five different samples (each using a different random number seed) for each porosity value. The edge length of the representative elementary volume (REV) is determined based on the sample porosity and equal to $11 d, 16 d$ and, $25 d$ for samples of mean porosities $79 \%, 85 \%$, and $95 \%$, respectively.

The fiber diameter is selected such that the fibers are optically large, i.e. the size parameter $x=\pi d / \lambda$ satisfies the condition $x>>1$, in the spectral range of interest, $\lambda=0.3-20 \mu \mathrm{m}$. Specifically, we select $x=100$. Since the clearance between the fibers, namely $c$, is of the same order of magnitude as the fiber diameter $d$, it 


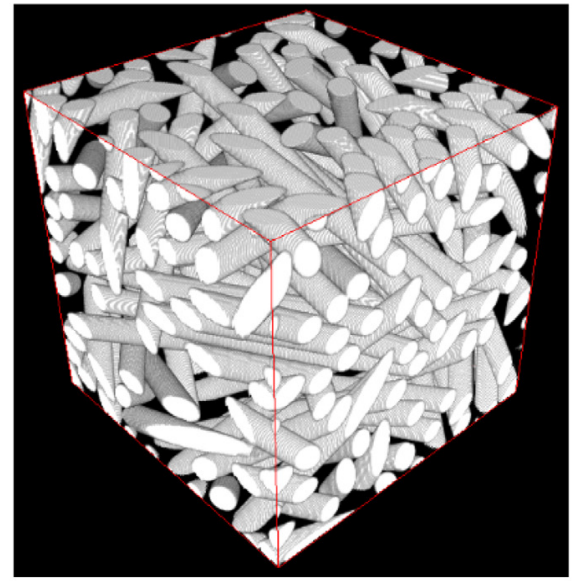

(a)

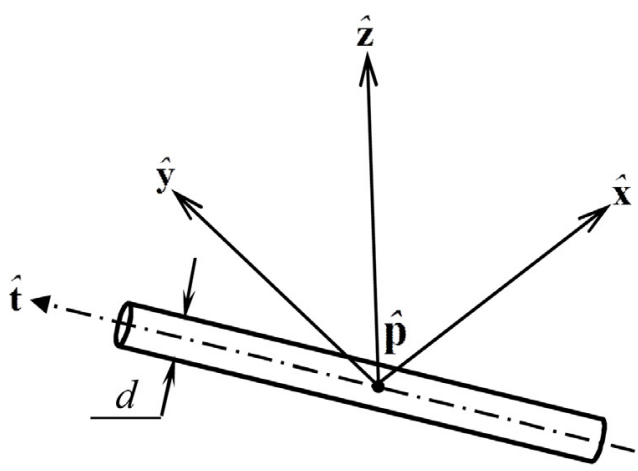

(b)

Fig. 1. (a) 3D rendering of the fibrous medium with porosity $85 \%\left(f_{v}=0.15\right)$, fiber diameter of $1 \mathrm{~mm}$ and edge length of $14.3 \mathrm{~mm}$. (b) Fiber geometrical characteristics: direction of fiber axis, $\hat{\mathbf{t}}$, point on the axis $\mathbf{p}$ in Cartesian reference, and diameter $d$.

follows that $c / \lambda>>1$. Thus, an electromagnetic wave incident on a fiber is assumed as planar, and the near-field radiative transfer and interference effects between scattered waves are neglected in the analysis. Consequently, geometric optics is employed to describe the interactions of electromagnetic waves with individual fibers, i.e. reflection, absorption, refraction, and diffraction. Since diffracted radiation has a strong forward peak in a narrow solid angle, it is assumed to be transmitted without any change of propagation direction. Destructive interference effects enhancing extinction inside optically large objects, as investigated by Berg et al. [37], are not considered.

The fibers are internally homogeneous, absorbing and nonscattering, with the spectral complex refractive index of the fiber material $m_{2, \lambda}=n_{2, \lambda}-i k_{2, \lambda}$. The host medium is homogeneous, nonrefractive and non-absorbing, $m_{1}=n_{1}=1$. The fibers are assumed to be optically smooth at the surface, i.e. Fresnel's equations [38] are used for the determination of the reflection and transmission characteristics.

\section{Methodology}

In order to predict macroscopic optical properties such as transmittances, reflectance and flux distributions from the knowledge of the fibrous materials, two approaches, namely the multi-RTE and the single-RTE approaches, are employed. Experimental data of macroscopic radiative quantities of the investigated fibrous materials are unavailable to our knowledge and reference results are obtained from the direct Monte Carlo simulation. Note that none of the methodologies is new. The multi-RTE approach has been initiated by Consalvi et al. [12] and developed by Lipinski et al. $[13,14]$. This method was derived by applying the volume averaging theorem by Whitaker and Quintard [39] to the multicomponent media. The single-RTE approach is based on traditional RTE $[1,2]$ along with appropriate properties of the equivalent homogeneous medium determined from the mean-free-path theory [40]. The latter assumes that local thermal equilibrium between components prevails. The DSMC applied to fibrous media is described in [32]. Here we present summaries of each method. The spectral subscript $\lambda$ is omitted for brevity. Equations used are solved on the spectral basis.

\subsection{Multi-RTE approach}

The change in intensity, $L$, in a radiative participating medium is described by the radiative transfer equation (RTE) [1,2]

$\hat{\mathbf{s}} \cdot \nabla L(\mathbf{x}, \hat{\mathbf{s}})=-\beta L(\mathbf{x}, \hat{\mathbf{s}})+n^{2} \kappa L_{\mathrm{b}}(\mathbf{x}, \hat{\mathbf{s}})+\frac{\sigma}{4 \pi} \int_{4 \pi} L\left(\mathbf{x}, \hat{\mathbf{s}}_{\text {in }}\right) \Phi\left(\hat{\mathbf{s}}_{\text {in }}, \hat{\mathbf{s}}\right) \mathrm{d} \Omega_{\text {in }}$

In a heterogeneous multi-component medium, one RTE per component needs to be solved [13]. In order to circumvent the complexity of the problem through the morphologically complex component structures, volume averaging approaches [39] can be used. They average the RTEs for each phase (air and fiber) in the limit of geometrical optics and introduce the appropriate discretescale boundary conditions at the phase interface. This leads to two coupled volume-averaged RTEs as described in $[12,13,17]$ and given by,

$$
\begin{aligned}
\hat{\mathbf{s}} \cdot \nabla I_{i}(\mathbf{x}, \hat{\mathbf{s}})= & -\beta_{i} I_{i}(\mathbf{x}, \hat{\mathbf{s}})+n_{i}^{2} \kappa_{i} I_{\mathrm{b}, i}(\mathbf{x}, \hat{\mathbf{s}}) \\
& +\frac{\sigma_{i i}}{4 \pi} \int_{4 \pi} I_{i}\left(\mathbf{x}, \hat{\mathbf{s}}_{\mathrm{in}}\right) \Phi_{i i}\left(\hat{\mathbf{s}}_{\mathrm{in}}, \hat{\mathbf{s}}\right) \mathrm{d} \Omega_{\text {in }} \\
& +\frac{\sigma_{j i}}{4 \pi} \int_{4 \pi} I_{j}\left(\mathbf{x}, \hat{\mathbf{s}}_{\text {in }}\right) \Phi_{j i}\left(\hat{\mathbf{s}}_{\mathrm{in}}, \hat{\mathbf{s}}\right) \mathrm{d} \Omega_{\text {in }} i, j=1,2 ; \mathrm{i} \neq \mathrm{j},
\end{aligned}
$$

where $I_{i}$ is the volume averaged intensity, $I_{i}=\frac{1}{V} \int_{V} L_{i} \mathrm{~d} V$, and

$\sigma_{i i}=\sigma_{\mathrm{refl}, i}+\sigma_{i}$

$\Phi_{i i}=\sigma_{i i}^{-1}\left(\Phi_{\mathrm{refl}, i} \sigma_{\mathrm{refl}, i}+\Phi_{i} \sigma_{i}\right)$

$\beta_{i}=\kappa_{i}+\sigma_{i i}+\sigma_{i j}=\beta_{\text {int }, i}+\sigma_{\text {refl }, i}+\sigma_{i j}$

$\beta_{\text {int }, i}=\kappa_{i}+\sigma_{i}$

The effective properties $\left(\sigma_{\mathrm{refl}, i}, \sigma_{i j}, \Phi_{\mathrm{refl}, i}\right.$, and $\Phi_{i j}$ ) provide the closure to the averaged equations, and are associated with internal reflection and refraction phenomena. Eqs. (2)-(6) are the general form of volume-averaged RTEs in heterogeneous two-component medium. Specifically, these equations take into account that the medium hosting the fibers and the fibers themselves may absorb and scatter radiation [41]. In that case, the radiation propagation in the phase $i$ (air or fiber substance) can be described by intrinsic radiative properties namely intrinsic extinction $\beta_{\text {int }, i}$, absorption $\kappa_{i}$, and scattering coefficients $\sigma_{i}$, and phase function $\Phi_{i}$. These intrinsic properties can be determined by measurements or applying an appropriate theory, e.g. Kerker [42,43]. For an non-absorbing phase (such as air) $\beta_{\text {int, } i}$ is zero. However, the effective extinction coefficient, $\beta_{i}$, of air component bounded by fiber surfaces is non-zero, where the interphase reflection (through $\sigma_{\text {refl, } i}$ and $\Phi_{\text {refl, } i}$ ) and refraction (through $\sigma_{i j}$ and $\Phi_{i j}$ ) phenomena contribute to the extinction. It is important to note that the intrinsic single phase radiative properties $\left(\beta_{\text {int }, i}, \kappa_{i}, \sigma_{i}\right.$ and $\left.\Phi_{i}\right)$ depend solely on the material 
properties of the phases involved. The two-phase radiative properties $\left(\sigma_{\text {refl, }, i}, \sigma_{i j}, \Phi_{\text {refl, } i}\right.$, and $\left.\Phi_{i j}\right)$ depend on morphology of the multi-phase media and its interface properties (specifically roughness and refractive indexes). In the present study, the two-phase radiative properties are derived from the corresponding probability distribution functions $[34,35]$ while they can be also derived by utilizing the mean-free-path theory applied to each phase [18].

The multi-scale radiation problem is solved in two steps. First, discrete-scale simulations are carried out at the discrete scale on the exact 3D microstructure described in Section 2 to determine

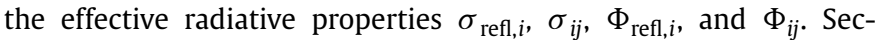
ond, these properties are incorporated in the volume-averaged system of Eq. (2), which are solved at the continuum scale to determine the macroscopic optical properties (e.g. radiative flux, transmittance and reflectance).

Geometrical optics are assumed since the characteristic size parameter satisfies the criterion $x=\pi \mathrm{d} / \lambda>>1[1,2,42]$. The refraction and reflection are modeled by employing Fresnel's equations and generalized Snell's law for an interface between an absorbing dispersed medium and a dielectric host medium (air in this current work) $[1,2,38]$.

The internal absorption coefficient of the fiber material is obtained using the relationship from the electromagnetic theory,

$\kappa_{2}=\frac{4 \pi k_{2}}{\lambda}$

The attenuation of radiative intensity inside a fiber is given by Bouguer's law,

$L=L_{0} \exp \left(-\kappa_{2} s\right)$

where $s$ is the path length inside the fiber.

The collision-based Monte Carlo ray-tracing method (MCRT) is used in combination with radiative distribution functions for semitransparent media $[7,14,17,36]$. A large number of stochastic rays $N$ distributed within REV of the fibrous samples are emitted. The manner to emit rays from within the REV is discussed in the next paragraph. Rays are then attenuated at the fluid-solid phase boundary and undergo either reflection or refraction. Fresnel's equations determine the probability for a ray to be reflected at the boundary. The generalized Snell's law [38] connects the incidence angle and the refraction angle. Additionally, rays are internally absorbed within the solid phase according to Eq. (8). The distance between emission and extinction points (for absorption or scattering), and the angle of incidence at the interface are recorded for each ray and contribute to the corresponding probability density functions. These statistical functions are directly related to the effective radiative properties of the two-phase medium $\sigma_{\text {refl, },}, \sigma_{i j}$, $\Phi_{\text {refl, },}$, and $\Phi_{i j}$ [34] and are input to the continuum-scale model calculations.

In the MCRT of effective properties calculations, the emission of the rays within the REV is defined in order to mimic as realistic as possible the propagation direction of rays within the sample. In a cold medium (which applies here when the volumetric emitted power is negligible compared to the boundary flux) with optically smooth fluid-solid phase boundaries (specular reflectivities), ray directions in the solid phase are constrained by the Snell's law at the phase interface (see Fig. 2). Rays could be thus distributed uniformly within the REV due to the random position of fibers and isotropically emitted in the fluid phase. However, in the solid phase, rays should be emitted within a solid angle oriented along the unit normal to the interface as depicted in Fig. 3. Indeed, all directions are not allowed within the fibers and ray directions depend on the refractive indexes of the fluid and solid phases. On the other hand, in an emitting fibrous medium or in the presence of irregular (or optically rough) phase boundaries (diffuse reflectivites), all ray directions are allowed in each phase so that rays

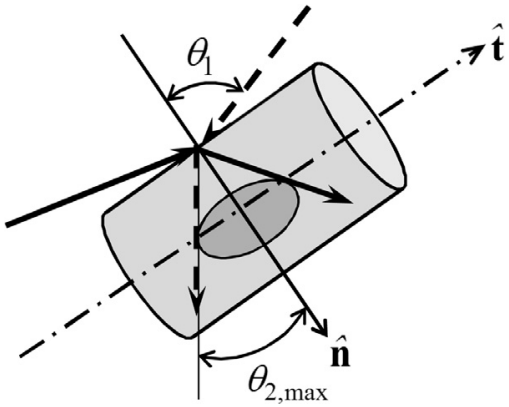

Fig. 2. Schematic depicting propagation of rays from the fluid phase to the solid phase. Rays in the solid phase are confined within a solid angle of characteristic angle $\theta_{2, \max }$ with $\theta_{2, \max }=\sin ^{-1}\left(n_{1, \lambda} \sin \theta_{1} / n_{2, \lambda}\right)$ when $k_{2, \lambda}<<n_{2, \lambda}$.

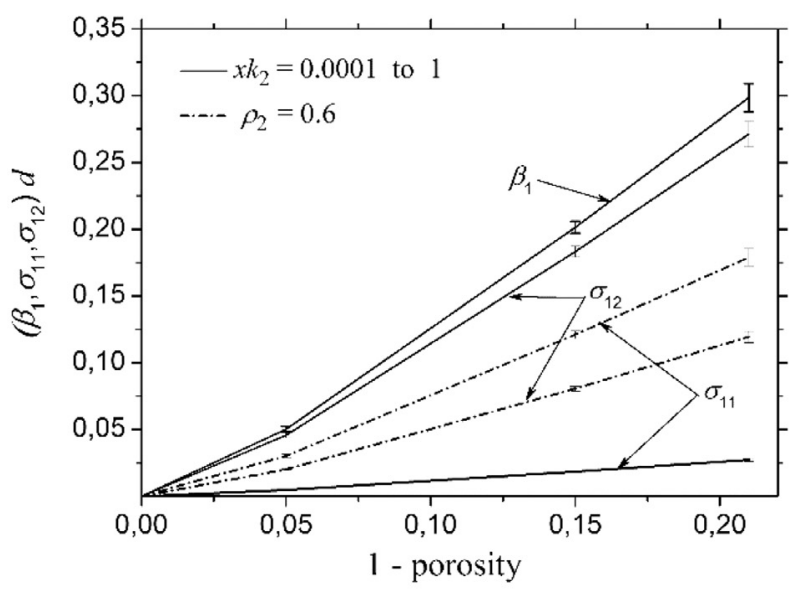

(a)

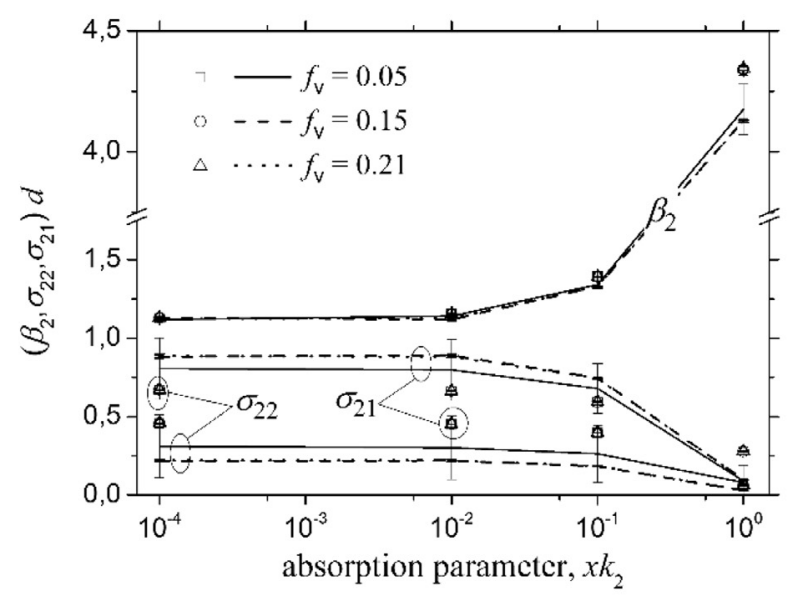

(b)

Fig. 3. Effective extinction and scattering coefficients (a) versus 1 - porosity in the fluid phase; and (b) versus the absorption parameter in the solid phase for emitting (symbols) and cold (curves) media; both for $n_{2} / n_{1}=1.5$ and $x=100$. The error bars in (a) and (b) indicate the standard deviation of coefficients based on computation over five different samples of identical size and porosity but generated with different random seeds.

can be uniformly distributed and isotropically emitted within the volume $V$ of the REV. The impact of the distribution of emission directions on effective radiative properties is discussed hereafter. It is worth noting that the effective properties computed in such ways are not systematically valid at boundaries of the medium due to near wall effects and the so-called near-wall radiative properties could be used [44]. However, due to the high porosity feature of 
the investigated fibrous samples, tests (not reported here) showed that the volumetric effective properties are suitable over the entire computational domain.

\subsection{Single-RTE approach}

In this approach, the two-phase fibrous medium is considered as a single homogeneous phase with effective radiative properties $\kappa_{\mathrm{a}}, \sigma_{\mathrm{a}}, \beta_{\mathrm{a}}=\kappa_{\mathrm{a}}+\sigma_{\mathrm{a}}$, and $\Phi_{\mathrm{a}}$ and the radiative transfer is modeled using Eq. (1). The radiative properties are calculated from the mean-free-path based method [40]. This approach is chosen among other due to its suitability to model properties of low and high porosity dispersed media consisting of optically large particles without assuming the independent scattering hypothesis. $[16,18,40]$

The method involves also the geometric optic hypothesis of the thermal radiation. Specifically, it computes the mean free path of extinction, $\Lambda$, the probability of scattering, $P_{\text {sca }}$, and the angular probability distribution of scattering within the two-phase medium, $P_{\text {sca }}(\Theta)$. The effective radiative properties are thus determined from these statistical parameters according to: [40]

$\beta_{\mathrm{a}}=\kappa_{\mathrm{a}}+\sigma_{\mathrm{a}}=\frac{1}{\Lambda}$

$\omega_{\mathrm{a}}=\frac{\sigma_{\mathrm{a}}}{\kappa_{\mathrm{a}}+\sigma_{\mathrm{a}}}=P_{\mathrm{sca}}$

$\Phi_{\mathrm{a}}(\Theta)=\frac{P_{\text {sca }}(\Theta)}{\frac{1}{4 \pi} \int_{0}^{4 \pi} P_{s c a}(\Theta) d \Omega}$

The MCRT simulation is also used to determine the quantities $\Lambda, P_{\text {sca }}$, and $P_{\text {sca }}(\Theta)$ involved in Eqs. (9)-(11). A large number of stochastic rays, $N$ (about $5 \times 10^{6}$ as discussed in 4.6), are emitted randomly from the external surfaces of fibers within the volume $V$ of the fibrous medium. The path of each ray is then followed from the emission point until it undergoes extinction. The extinction takes place when the ray (i) reflects at the fluid phase-fiber interface; (ii) is absorbed inside a fiber; or (iii) crosses a fiber after one or several interior travels. The distance traveled by the ray from the emission point to the extinction point is computed and stored. Additionally, when a scattering occurs, the angle between the incident direction of the ray and the direction of the ray after the scattering, namely $\Theta$, is determined. The number of rays scattered in a given angular interval $\Theta$ and $\Theta+\mathrm{d} \Theta$ is incremented. Finally, the mean free path of extinction $\Lambda$ is given by the arithmetic mean of extinction distances of $N$ rays. The scattering probability $P_{\text {sca }}$ is computed as the fraction of $N$ rays undergoing scattering. The directional probability distribution of scattering $P_{\text {sca }}(\Theta)$ is obtained as the ratio of rays scattered in a given angular interval $\Theta$ and $\Theta+\mathrm{d} \Theta$ to the number of rays undergoing scattering. More details about the MCRT applied to other disperse media can be found elsewhere $[18,40]$.

In this study, we specifically consider the case of non-refracting and non-attenuating host medium (air). However, the single-RTE approach could be also suitable to a refractive and weakly absorbing medium surrounding the fibers. The absorption in the host medium can be included through the calculation of effective radiative properties as it was done for the radiative transfer in bed of spherical particles in a refracting and weakly absorbing host medium. [40] The case of particles in a moderately to strongly absorbing medium cannot be modeled by either the present single or multi-RTE approach. The reader is referred to the pertinent literature, e.g. [45-47].

\subsection{Macroscopic radiative characteristics calculations}

\subsubsection{Multi-RTE approach}

A slab with a certain thickness, namely $L_{\text {slab }}$, and infinite extends in the other two dimensions is used as the 3D computational domain for the determination of the macroscopic radiative characteristics, i.e. radiative flux, reflectance and transmittance. The slab is exposed to a collimated or a diffuse radiative flux $q_{\text {in }}$. To model the infinite extends in the other two dimensions of the slab, a periodic boundary conditions is applied. Specifically, when a radiation beam reaches one of the lateral edges of the slab (i.e. the four boundaries parallel to the thickness direction), it is allowed to undergo mirror-like reflexion. The inlet and outlet faces are exposed to a black cold surroundings. Additionally, we accounted for the inlet and outlet boundary effects between the semi-transparent phase (fiber) and the surrounding (see Appendix for details). Pathlength Monte Carlo [48] is used to solve the two coupled volumeaveraged RTEs, Eq. (2), using the appropriately determined effective radiative properties. The overall radiative flux distribution along the $z$-axis is defined by:

$\bar{q}(z)=\frac{\bar{q}_{1}(z)+\bar{q}_{2}(z)}{q_{\text {in }}} \quad 0<z<L_{\text {slab }}$,

with $\bar{q}_{i}$ the radiative flux averaged over the cross-sectional area in forward and backward directions and in the void or solid phase (i: 1, 2).

The overall reflectance $R$ and transmittance $\operatorname{Tr}$ are respectively inlet $(z=0)$ and outlet $\left(z=L_{\text {slab }}\right)$ area-averaged, and defined by:

$R=\frac{\bar{q}_{1}^{-}(z=0)+\bar{q}_{2}^{-}(z=0)}{q_{\text {in }}}$,

$\operatorname{Tr}=\frac{\bar{q}_{1}^{+}\left(z=L_{\text {slab }}\right)+\bar{q}_{2}^{+}\left(z=L_{\text {slab }}\right)}{q_{\text {in }}}$,

In Eqs. (13) and (14), $\bar{q}_{i}^{j}$ refers the radiative flux averaged over the cross-sectional area in forward or backward direction $(j:+,-)$, and in the void or solid phase $(i: 1,2)$. Thus, $R$ and $T r$ are the fractions of incident radiative flux leaving the slab at $z=0$ and $z=L_{\text {slab }}$ respectively. The radiative fluxes are computed from the history of rays crossing any surface of abscissa $z$ and unit normal along the $z$-axis.

\subsubsection{Single-RTE approach}

The traditional collision based MC method described in standard textbooks $[1,2,48]$ is used to solve the single homogeneous phase RTE, Eq. (1), using the computed radiative properties $\kappa_{\mathrm{a}}, \sigma_{\mathrm{a}}$, $\beta_{\mathrm{a}}$ and $\Phi_{\mathrm{a}}$ from the mean-free-path method. Infinite slabs are considered so that the sample is only characterized by the slab thickness $L_{\text {slab }}$. The boundary conditions are identical to those considered in the multi-RTE approach, namely (i) a heat flux $q_{\text {in }}$ is applied on the inlet face of the slab at $z=0$; and (ii) the inlet and outlet faces are exposed to black cold medium. The reflection of rays by fiber slices at inlet is implemented in the continuum calculations by using a probability of reflection at the inlet face defined as $f_{\mathrm{s}} \rho$. Here $f_{\mathrm{s}}$ and $\rho$ refer respectively to the surface fraction of fiber slices on the inlet face and the reflectivity of fiber. The latter parameter is a function of optical constants of the fiber and the surrounding medium through Fresnel's relations (see also Appendix for more details). The apparent radiative flux along the $z$-axis is defined by:

$q_{a}=\frac{q^{-}(z)+q^{+}(z)}{q_{\text {in }}} \quad 0<z<L_{\text {slab }}$,

The MC simulation provides the histories of rays throughout the sample. Thus, the reflectance and transmittance are computed 
from the fractions of rays leaving the slab from the inlet face and the outlet face:

$R=\frac{q^{-}(z=0)}{q_{\text {in }}}$,

$\operatorname{Tr}=\frac{q^{+}\left(z=L_{\text {slab }}\right)}{q_{\text {in }}}$

\subsubsection{Direct simulation Monte Carlo method}

The reference data of macroscopic radiative properties are obtained from DSMC of radiation propagation through 3D fibrous slabs described in Section 2. It simulates the propagation of the incident flux $q_{\text {in }}$ from the inlet through the fibrous slab. For simplicity, the medium is assumed to be cold so that self-emission could be neglected. The inlet and outlet faces are also exposed to black cold medium while periodic boundary conditions are implemented at other boundaries of the computational volume $V$. The incident radiation flux is subdivided into $N$ independent rays. The DSMC algorithm is then applied to follow the path of each ray within the two-phase discrete medium. The reflections of rays at the inlet and outlet faces when they interact with the surrounding medium - fiber and fiber - surrounding medium interfaces are accounted for, respectively. The track of the ray path is terminated when either it leaves the slab from inlet and outlet boundaries at $z=0$ and $z=L_{\text {slab }}$, respectively, or it is absorbed inside a fiber. The number of rays over cross-sectional area in the forward and backward directions and the number of rays leaving the slab from the inlet and the outlet, are again used to compute the radiative flux, reflectance and transmittance through Eqs. (11)-(14). More details about the DSMC to simulate light propagation in slabs of optically large fibers with random orientation can be found elsewhere. [32]

\section{Results}

\subsection{Material parameters}

The radiative transfer in fibrous slabs having three different porosities of $95 \%, 85 \%$, and $79 \%$ is analyzed. Fibers exhibit different complex refractive indexes of uniform real part, $n_{2}=1.5$ and imaginary part, $k_{2}$, of $0.01,0.001,0.0001$, and 0.000001 . For mimicking fibrous media of opaque reflecting fibers, fibers with uniform reflectivity equal to $\rho_{2}=0.6$ and very large imaginary part of complex refractive, $k_{2}=+\infty$, are considered. To ensure meaningful macroscopic radiative characteristics (i.e. results larger than the associated uncertainty), moderate slab thicknesses $L_{\text {slab }}$ are considered and they are thus different from one porosity to another. Specifically $L_{\text {slab }} / d$ values are 25,8 and 5.833 for porosities $95 \%$, $85 \%$, and $79 \%$, respectively.

\subsection{Effective radiative properties of the multi-RTE approach}

Fig. 3(a) shows the evolution of the normalized, effective extinction $\beta_{1}$ and scattering coefficients for the fluid phase $\sigma_{11}$ and $\sigma_{12}$ versus 1 - porosity. Since the medium surrounding the fiber is transparent, the dimensionless effective extinction coefficient of the fluid phase defined as $\beta_{1} \times d$ increases with the fiber volume fraction. The relationship between effective extinction coefficient and porosity is not linear. This is in contrast to the linear relationship between extinction and porosity stated by the independent scattering theory (i.e. $\beta_{\text {ind }}$ varies as $Q_{\text {ext }} f_{\mathrm{v}} / d$ with $Q_{\text {ext }}$ the extinction efficiency factor of an isolated fiber [30]). The effective scattering coefficients have also the same trend as the effective extinction coefficient but they can be dependent on optical properties of the solid phase. For a medium of reflecting opaque fibers and a given porosity, $\sigma_{11}$ is greater than $\sigma_{12}$ due to the high value of the fiber surface reflectivity ( $\rho_{2}=0.6$ here). Inversely, $\sigma_{12}$ is systematically greater than $\sigma_{11}$ for all other samples since they have small surface reflectivity ( $\rho_{2}$ about 0.04 for near normal incidence of rays onto the fluid-solid surface and for $n_{2} / n_{1}=1.5$ ). The effective scattering coefficient $\sigma_{11}$ or $\sigma_{12}$ is similar for all values of the parameter $x k_{2}$ due to negligible effect of the fiber phase absorption index $k_{2}$ on the Fresnel's coefficients at the air-fiber interface since $k_{2}<<n_{2}$ [38].

Fig. 3(b) reports the corresponding effective coefficients for the solid phase $\beta_{2}, \sigma_{22}$ and $\sigma_{21}$ versus the fiber absorption parameter $x k_{2}$ ranging from $10^{-4}$ to 1 . Both the effective coefficients of an emitting medium and a cold fibrous medium are analyzed. Given that the radiative transfer within opaque solid phase (case of $x k_{2}>>1$ ) is negligible, the analysis of their effective coefficients are of no interest. We can observe first that the impact of the fiber volume fraction on effective coefficients is insignificant. This is only true because the fibers do not overlap with each other. In the limit of cold medium (and with regular solid-fluid phase boundaries), the effective coefficient $\sigma_{21}$ is greater than the effective coefficient $\sigma_{22}$. It means the transmission of rays from the solid phase to the fluid phase is more effective than reflexion of rays to the solid phase. We observe the inverse trend with an emitting medium (or with irregular phase boundaries), i.e. $\sigma_{22}$ is greater than $\sigma_{21}$ at any value of $x k_{2}$. The effective coefficients change slightly with the solid phase absorption as long as the parameter $x k_{2}$ is less than 0.1 (which can be referred to as the limit of weakly absorbing fibers). They then change sensibly for values of $x k_{2}$ above 0.1. Specifically, fibers with $x k_{2}$ of 1 lead to effective coefficients $\sigma_{21}$ and $\sigma_{22}$ approaching zero and $\beta_{2}$ about $4 / d$ to $4.3 / d$. Note that this range of $\beta_{2}$ is close to the intrinsic (or bulk) absorption coefficient of the solid phase $\kappa_{2}\left(=4 x k_{2} / d\right)$. The value of $x k_{2}=1$ thus corresponds to the limit of absorbing fibers where absorption controls extinction.

Fig. 4(a)-(d) report the set of effective scattering functions and the corresponding effective asymmetry factors for the fibrous medium of $85 \%$ porosity. They are representative of the two other fibrous media of $95 \%$ and $79 \%$ porosities. In the fluid phase, the scattering of rays by reflection and by transmission are globally forward expect for opaque fibers where the scattering by reflection is isotropic ( $\Phi_{11}=1$ and $\left.g_{11}=0\right)$. This last is a well-known scattering behavior of opaque particles with regular boundaries (specular surface reflectivities) [1,2]. In the solid phase, the scattering by transmission is highly forward ( $g_{21}>0.9$ over the range of fiber absorption index $\left.10^{-4} \leq x k_{2} \leq 1\right)$ and the effect of the type of the medium (e.g. cold or emitting medium) on angular scattering distribution is moderately small. However, the angular distribution of scattering by reflection in the solid phase is strongly altered by the type of the medium. A cold fibrous medium with regular solidfluid phase boundaries features a scattering function exhibiting an unusual peak in the direction close to the normal to the direction of incident rays. The exact location of this peak depend on refractive indexes of the solid and fluid phases and is actually at a scattering angle of $97^{\circ}$ for $n_{2} / n_{1}=1.5$. The peak in $\Phi_{22}$ corresponds to reflection of rays confined within the solid angle of propagation (as depicted in Fig. 2) at the internal fiber surface. An emitting fibrous medium exhibits a scattering function $\Phi_{22}$ in forward directions. The more the solid phase absorbs the more forward the scattering is. Note that this scattering behavior is a known characteristic of two-phase media in the geometric optic limit and with irregular phase boundaries (e.g. in [7]).

\subsection{Effective radiative properties of the single-RTE approach}

Fig. 5(a) shows the extinction coefficient versus 1-porosity. For comparison, we report also the extinction coefficient predicted by the independent scattering theory, $Q_{\text {ext }} f_{\mathrm{v}} / d$ with $Q_{\text {ext }}$ equal to 1 in the limit of optically large fiber $(x>>1)$ and without diffrac- 


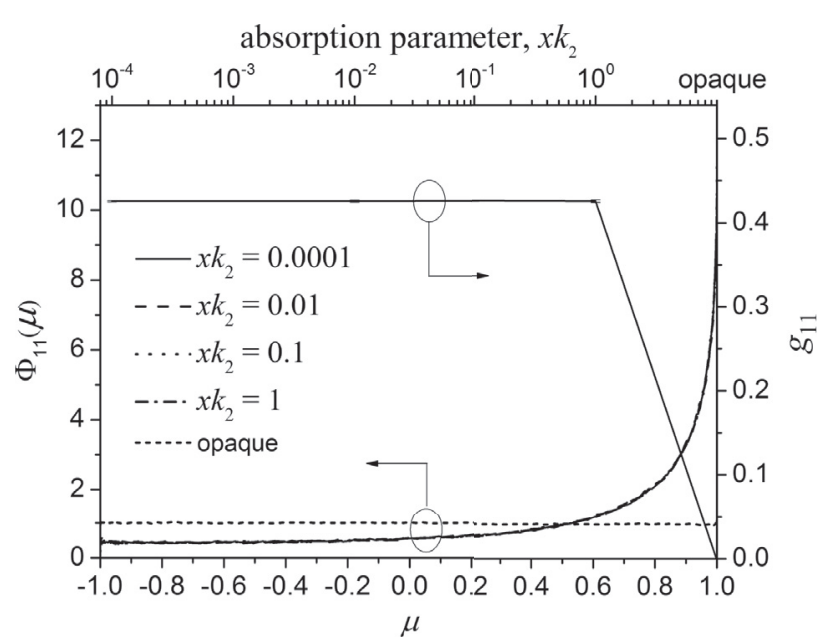

(a)

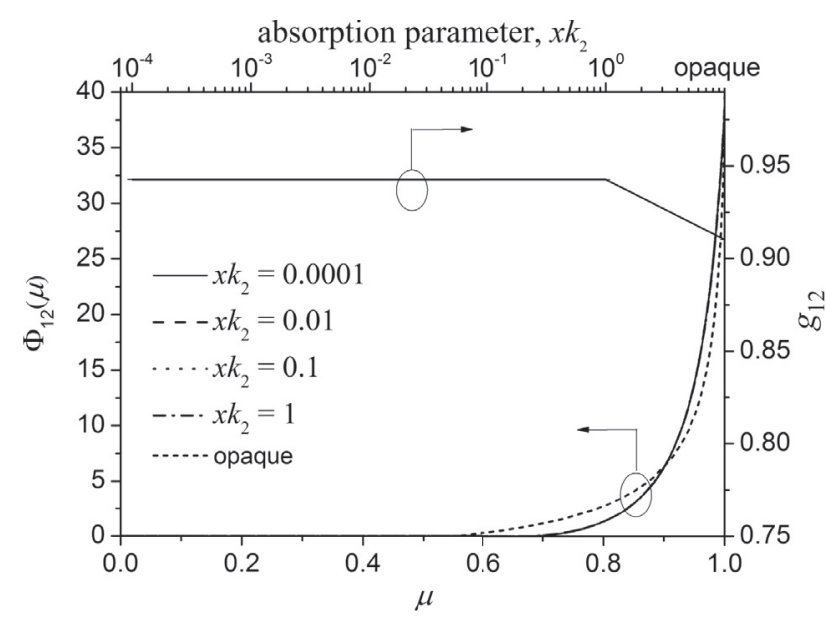

(b)

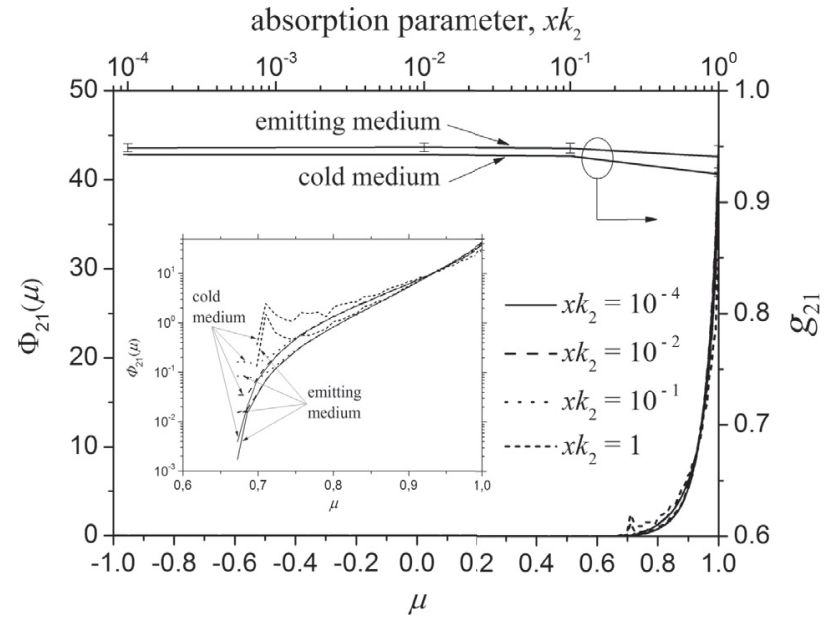

(c)

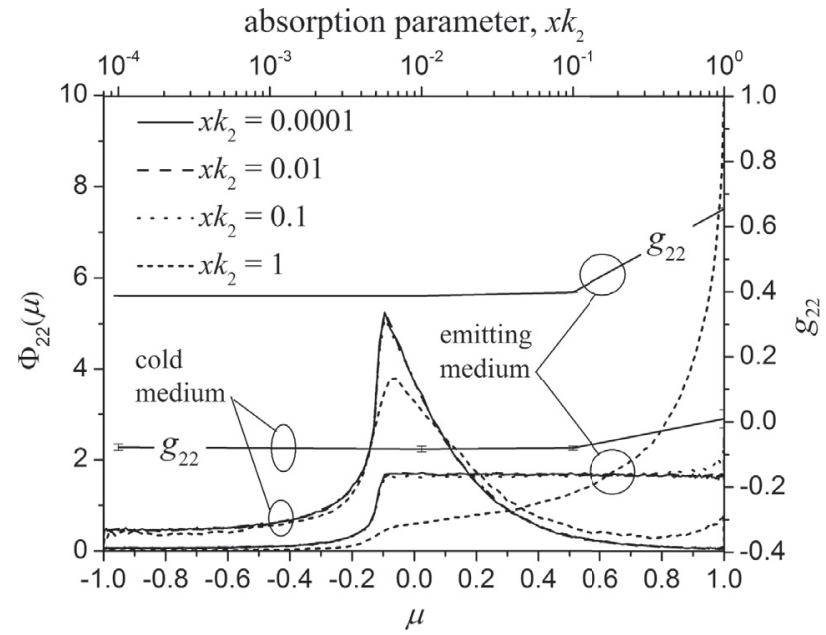

(d)

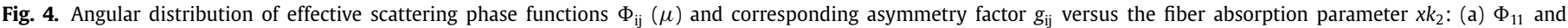
$g_{11}$; (b) $\Phi_{12}$ and $g_{12}$; (c) $\Phi_{21}$ and $g_{21}$; and (d) $\Phi_{22}$ and $g_{22}$; all for $n_{2} / n_{1}=1.5$ and $x=100$.

tion contribution [30]. We can see that for porosities greater than $95 \%$, the apparent extinction coefficient varies linearly with (1porosity) and is independent of the fiber absorption characteristic. The results from the mean-free-path and independent scattering approaches are close to each other. For a porosity value less than $95 \%$, more absorbing the fibers, larger the extinction coefficient is indeed, weakly absorbing fibers allow internal travels of rays and thus a longer extinction mean free path (or a smaller extinction coefficient) than opaque or highly absorbing fibers [40]. The deviation of extinction coefficient computed from the mean-free-path approach and that predicted by independent scattering theory increases with the fiber volume fraction or (1-porosity). The reason of this deviation is attributed to the breakdown of the point scattering hypothesis involved in the independent scattering theory. As the porosity decreases, the clearance between fibers becomes comparable to the fiber size so that the fibers cannot be viewed as point scatterers.

The scattering coefficient $\sigma_{\mathrm{a}}$ versus ( 1 - porosity) is shown in Fig 5(b). The scattering coefficient varies with the porosity in the same manner as the extinction coefficient reported in Fig 5(a). The impact of the fiber absorptivity on the scattering coefficient is clearly noticeable. The more absorbing the fibers, the lower their scattering efficiency is. Scattering by the fibrous medium could consist of surface reflection and refraction. In the limit of highly absorbing fibers $\left(x k_{2} \geq 1\right)$, scattering is by surface reflection (diffraction is omitted) and is reinforced by the non-point scattering effect due to non-vanishing volume of fibers at high volume fraction. [49,50] For fibers of refractive index ratio $n_{2} / n_{1}=1.5$ and absorption index $k_{2}<<n_{2}$ surface reflectivity is small (hemispherical reflectivity about $10 \%$ ) and as a result the scattering coefficient is also small. However, for the opaque fibers of surface reflectivity $\rho_{2}=0.6$ scattering coefficient is important especially at low porosity.

Fig. 5(c) and (d) report the scattering phase functions for the four different optical properties of fibers and the corresponding asymmetry factors, respectively. The scattering functions of weakly absorbing fibers $\left(x k_{2}<1\right)$ are qualitatively similar and feature a strong forward peak $\left(g_{a}>0.6\right)$ due to transmission of incident rays across the fibers. As the fiber becomes more absorbing $\left(x k_{2}=1\right)$, transmission of rays across the fibers leading to the forward scattering peak decreases while external surface reflection of rays remains present. As result, the scattering tends to be isotropic. For opaque fibers, the scattering function is only due to external surface reflection of rays. For randomly oriented fibers with uniform surface reflectivity the scattering is isotropic $\left(g_{a}=0\right)$ in accordance to the theory of optically large opaque particles. $[1,2]$ 


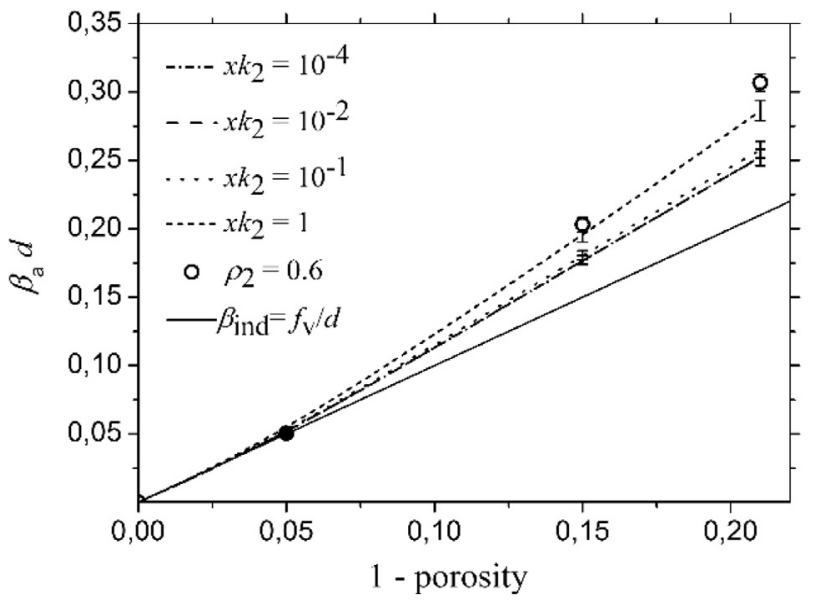

(a)

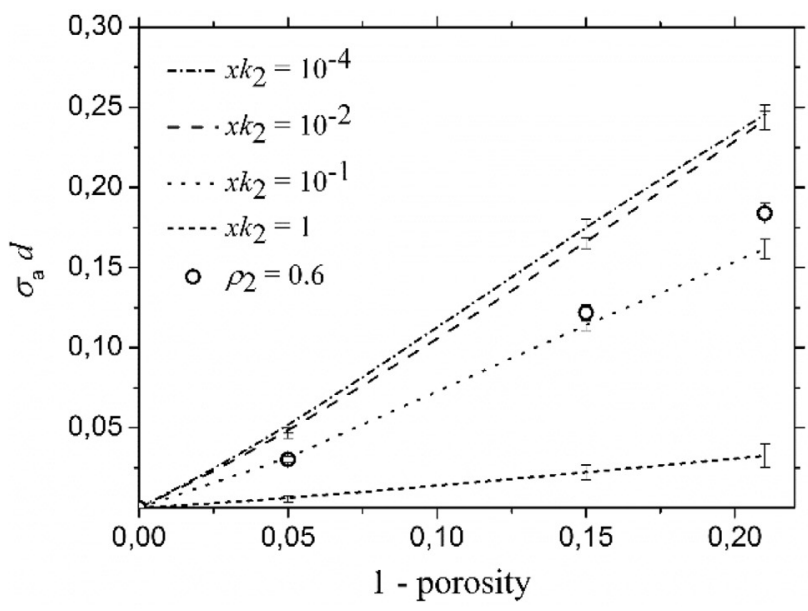

(b)

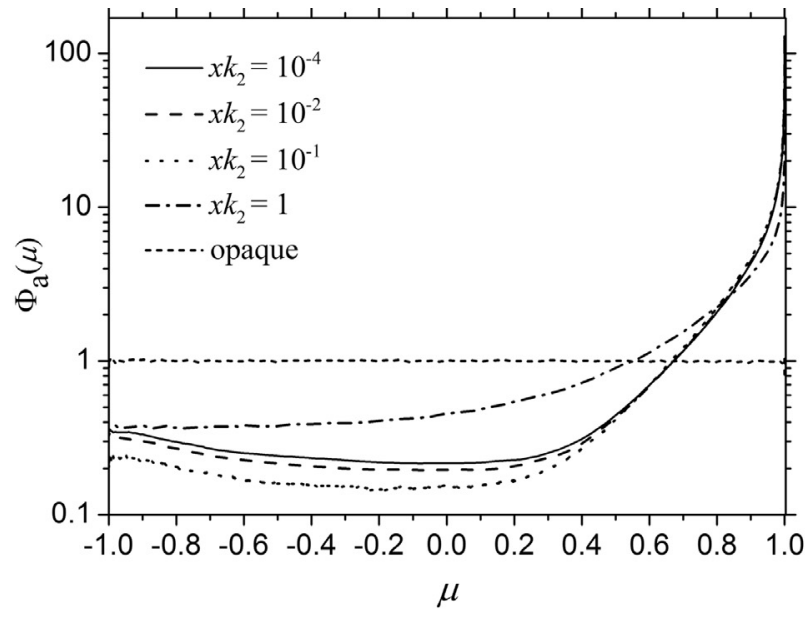

(c)

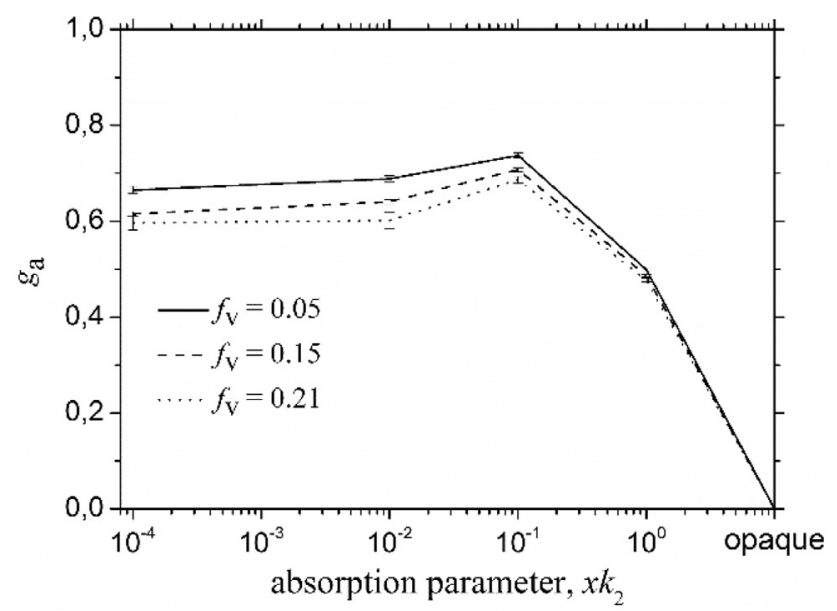

(d)

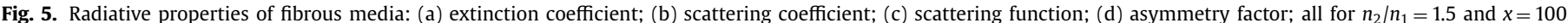

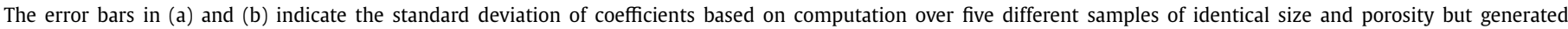
with different random seeds.

Table 1

Transmittanes, $T r$, and Reflectances, $R$, of fibrous medium with $95 \%$ of porosity subjected to a collimated incident radiative flux with $L_{\text {slab }} / d=25$.

\begin{tabular}{|c|c|c|c|c|c|c|}
\hline \multirow[t]{2}{*}{ Absorption parameter $\mathrm{xk}_{2}$} & \multicolumn{2}{|c|}{ Single-RTE approach } & \multicolumn{2}{|c|}{ Multi-RTE approach } & \multicolumn{2}{|c|}{ Reference DSMC } \\
\hline & $\overline{T r}$ & $R$ & $T r$ & $R$ & $T r$ & $R$ \\
\hline $10^{-4}$ & 0.864 & 0.1340 & 0.859 & 0.1399 & 0.897 & 0.1014 \\
\hline $10^{-2}$ & 0.813 & 0.1014 & 0.821 & 0.1196 & 0.839 & 0.0803 \\
\hline 0.1 & 0.612 & 0.0352 & 0.611 & 0.0447 & 0.614 & 0.0310 \\
\hline 1 & 0.353 & 0.0084 & 0.334 & 0.0088 & 0.397 & 0.0081 \\
\hline$+\infty$ & 0.409 & 0.1550 & 0.398 & 0.1483 & 0.356 & 0.1624 \\
\hline
\end{tabular}

\subsection{Transmittance and reflectance}

\subsubsection{Slabs subjected to a collimated incident flux}

Tables 1-3 report the transmittances and reflectances of three fibrous slabs of $95 \%, 85 \%$ and $79 \%$ porosities subjected to a collimated incident radiative flux. The results obtained from the two investigated approaches and the reference method present globally the same trends. To be consistent with cold medium assumption used in the reference DSMC method, the effective radiative properties involved in the multi-RTE approach are those corresponding to cold medium with regular phase boundaries. For a fixed porosity, transmittance and reflectance decrease when the value of fiber ab- sorption index $k_{2}$ increases or in another word, when the medium becomes more and more absorbing. For a fixed value of the fiber absorption index and a decreasing value of porosity, the transmittance gets smaller while the reflectance increases. This is because the lower the porosity, the smaller the fraction of directly transmitted radiation and stronger scattering within the bed are. For the sample of $95 \%$ porosity, the prediction from the two approaches are close to each other. The agreement between the two approaches and the reference DSMC is also satisfying: the largest relative errors with the multi- and single-RTE approaches (respectively about $33 \%$ and $24 \%$ ) are on small magnitude of reflectances for values of $x k_{2}$ equal to 0.1 and 1. For samples of $85 \%$ and $79 \%$ 
Table 2

Transmittanes, $\operatorname{Tr}$, and Reflectances, $R$, of fibrous medium with $85 \%$ of porosity subjected to a collimated incident radiative flux with $L_{\text {slab }} / d=8$.

\begin{tabular}{|c|c|c|c|c|c|c|}
\hline \multirow[t]{2}{*}{ Absorption parameter $x k_{2}$} & \multicolumn{2}{|c|}{ Single-RTE approach } & \multicolumn{2}{|c|}{ Multi-RTE approach } & \multicolumn{2}{|c|}{ Reference DSMC } \\
\hline & $\operatorname{Tr}$ & $R$ & $\operatorname{Tr}$ & $R$ & $\operatorname{Tr}$ & $R$ \\
\hline $10^{-4}$ & 0.809 & 0.1887 & 0.792 & 0.1767 & 0.785 & 0.1743 \\
\hline $10^{-2}$ & 0.751 & 0.1430 & 0.781 & 0.1715 & 0.768 & 0.1651 \\
\hline 0.1 & 0.534 & 0.0505 & 0.505 & 0.0597 & 0.469 & 0.0609 \\
\hline 1 & 0.267 & 0.0169 & 0.202 & 0.0155 & 0.175 & 0.0163 \\
\hline$+\infty$ & 0.278 & 0.2663 & 0.245 & 0.2485 & 0.212 & 0.2524 \\
\hline
\end{tabular}

Table 3

Transmittanes, $T r$, and Reflectances, $R$, of fibrous medium with $79 \%$ of porosity subjected to a collimated incident radiative flux with $L_{\text {slab }} / d=5.833$.

\begin{tabular}{|c|c|c|c|c|c|c|}
\hline \multirow[t]{2}{*}{ Absorption parameter $x k_{2}$} & \multicolumn{2}{|c|}{ Single-RTE approach } & \multicolumn{2}{|c|}{ Multi-RTE approach } & \multicolumn{2}{|c|}{ Reference DSMC } \\
\hline & $\operatorname{Tr}$ & $R$ & $\operatorname{Tr}$ & $R$ & $\operatorname{Tr}$ & $R$ \\
\hline $10^{-4}$ & 0.762 & 0.2359 & 0.758 & 0.2398 & 0.742 & 0.2560 \\
\hline $10^{-2}$ & 0.694 & 0.1804 & 0.698 & 0.2029 & 0.632 & 0.2139 \\
\hline 0.1 & 0.466 & 0.0675 & 0.404 & 0.0743 & 0.383 & 0.0781 \\
\hline 1 & 0.204 & 0.0271 & 0.127 & 0.0194 & 0.119 & 0.0199 \\
\hline$+\infty$ & 0.194 & 0.3219 & 0.157 & 0.3046 & 0.140 & 0.2876 \\
\hline
\end{tabular}

porosities, the multi-RTE approach is clearly superior to the singleRTE approach. Indeed, the former and latter approaches feature respectively maximum relative errors about $13 \%$ and $41 \%$ at high values of $x k_{2}(>1)$. Though significant, the relative error of the multi-RTE approach is comparable to the uncertainties of reference method (about 10-15\%), mainly due to statistical non-homogeneity of samples as discussed in Section 4.6.

\subsubsection{Slabs subjected to a diffuse incident flux}

Tables 4-6 summarize the transmittances and reflectances of fibrous slabs described in Section 4.1. Here, the samples are exposed to diffuse incident flux on inlet boundary $z=0$. The trend and magnitude order of transmittances and reflectances are similar to the case of slabs subjected to a collimated incident flux so that the conclusion in Section 4.4.1 holds. However, the values of transmittance are reduced due to the small amount of radiation traveling straight path through the slab in the presence of diffuse irradiation. The results from the two investigated approaches are in qualitative agreement with the reference data given that their absolute deviations remain small. At the same time, the relative errors are important as low values of transmittances and reflectances are concerned. The largest relative error of the multi-RTE approach is about $25 \%$ on reflectance of the sample of $95 \%$ porosity and absorption parameter $x k_{2}=0.1$ (see Table 4). Concerning the singleRTE approach, the relative error reaches $48 \%$ on transmittance of the sample of $79 \%$ porosity and absorption parameter $x k_{2}=1$ (see Table 6). It is clear from this comparison that the multi-RTE approach is suitable to predict transmittances and reflectances of the investigated fibrous slabs irradiated on one face with a diffuse flux.

\subsection{Radiative flux}

For the analysis of radiative flux distribution, we focus our attention on the case of sample subjected to collimated flux since the following conclusion is globally valid also for cases of diffuse incident flux. Figs. 6-8 depict the radiative flux distribution along the slab thickness for the porosity values from $95 \%$ down to $79 \%$ and for the four different fiber absorption parameters. The heat flux is maximal near the inlet as results of the incident radiation flux. It then gradually decreases through the slab depth and is minimal near the outlet boundary where the forward flux tends to equal the backward one. At high porosity value (Fig. 6), the results from the two modeling approaches match well the DSMC data ex- pect the case of weakly absorbing slab (Fig. 6(a)) where the singleRTE approach underestimates the radiative flux. The suitability of the single-RTE approach is not surprising here, since at high porosity the contribution of radiative transfer within the fiber phase to the macroscopic characteristics is small compared to that within the air and thus, the traditional continuum radiative transfer equation with effective properties is valid. As the porosity decreases (Figs. 7 and 8), the single-RTE approach fails while the multi-RTE approach remains globally accurate. This is consistent with results of transmittances and reflectances (Tables 2 and 3). The weakness of the single-RTE approach could be explained by two reasons. At low porosity, the radiative transfer within the fiber phase contributes meaningfully to the macroscopic characteristics but this is not accounted for in the single-RTE approach. The second reason comes probably from the effect of the finite length of fibers that is not taken into account in the radiative properties. Indeed, the radiative properties calculation in the single-RTE approach assumes that the fibers are infinitely long while in the DSMC, they have finite length (the length to diameter ratios $L / d$ are about 6 and 8 for $79 \%$ and $85 \%$ porosities, respectively). In the multi-RTE approach, the finite length of the fibers is accounted for through the boundary conditions coupled to the volume-averaged radiative equations. A ray reaching the inlet or outlet boundary within the solid phase could be reflected or transmitted out but this phenomenon is neglected in apparent radiative transfer approach. Although globally satisfying, it is worth noticing that the multi-RTE approach shows some difficulties to model correctly the radiative flux distribution of weakly absorbing fibrous media (Figs. 7(b) and 8(a)).

\subsection{Numerical implementation and accuracy consideration}

The computational conditions in term of the ray number, $N$, REV size and numerical implementation were keep identical for each approach. Specifically, the three computational approaches were implemented in Intel Fortran 2013 along with the message passing interface (MPI) protocol allowing parallel computing. The simulation was performed using multiple core computers (Bull R421E3 Cluster, Intel Xeon Processors E5-2650v2 8C 2.600 GHz 32 Go DDR3, InfiniBand fast network FDR [51] and IBM cluster, AMD Opteron 6176 24C $2.300 \mathrm{GHz} 24$ Go RAM, InfiniBand fast network QDR [52]). Fig. 9 shows typical computation time per CPU versus the fiber absorption parameter $x k_{2}$ of the single and multiRTE approaches for $95 \%$ and $79 \%$ porosity samples. Samples with 


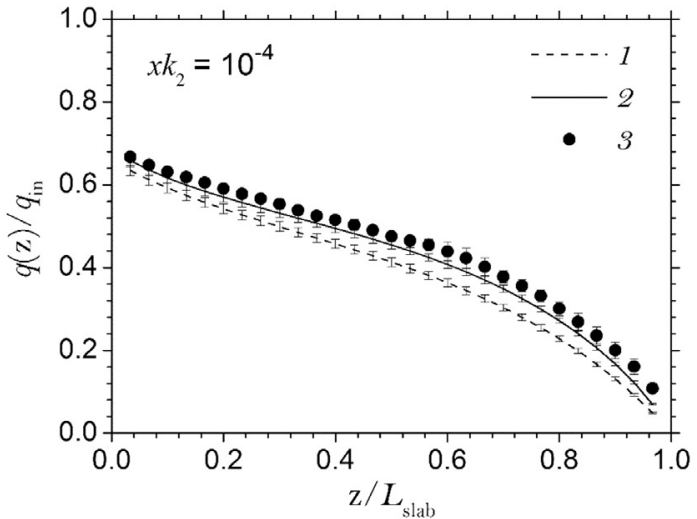

(a)

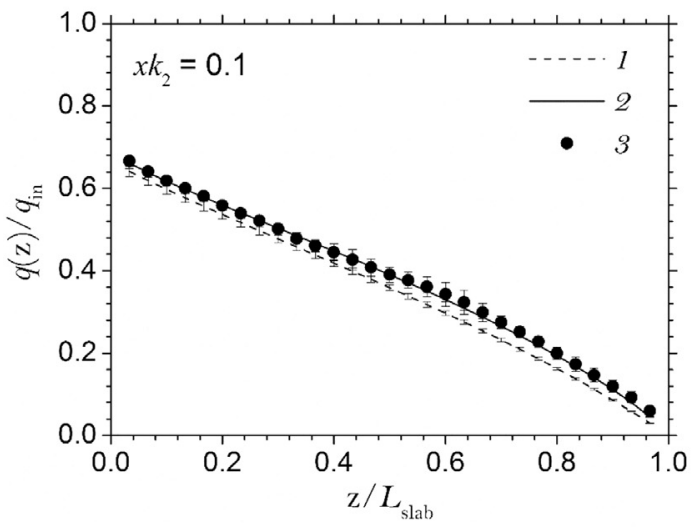

(b)

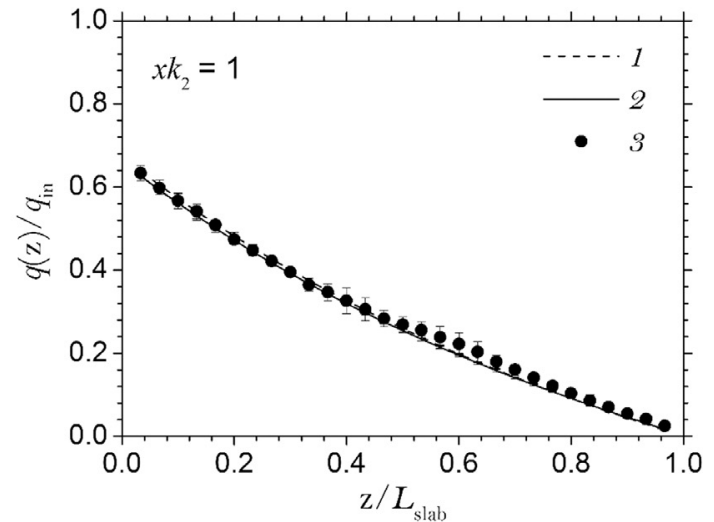

(c)

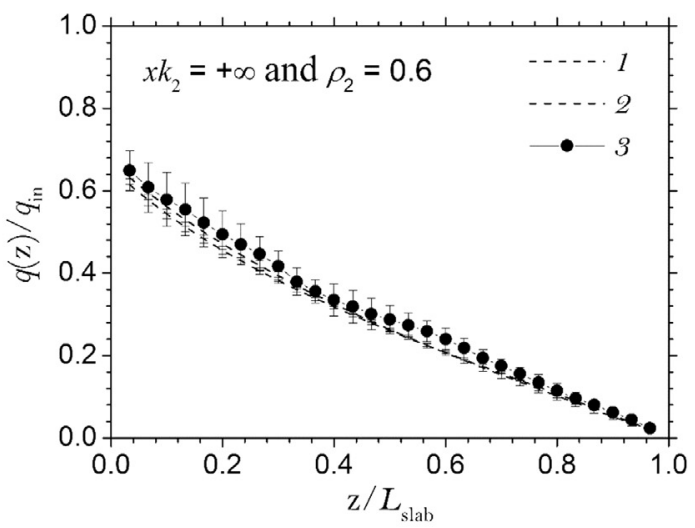

(d)

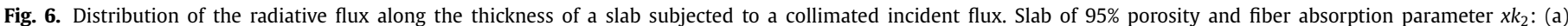

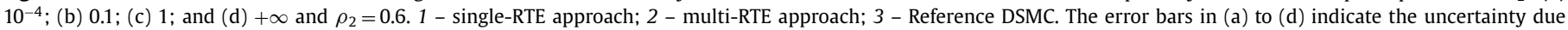

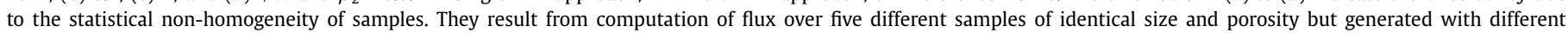
random seeds.

Table 4

Transmittances, $T r$, and Reflectances, $R$, of fibrous medium with $95 \%$ of porosity subjected to a diffuse incident radiative flux with $L_{\text {slab }} / d=25$.

\begin{tabular}{|c|c|c|c|c|c|c|}
\hline \multirow[t]{2}{*}{ Absorption parameter $x k_{2}$} & \multicolumn{2}{|c|}{ Single-RTE approach } & \multicolumn{2}{|c|}{ Multi-RTE approach } & \multicolumn{2}{|c|}{ Reference DSMC } \\
\hline & $\operatorname{Tr}$ & $R$ & $\operatorname{Tr}$ & $R$ & $\operatorname{Tr}$ & $R$ \\
\hline $10^{-4}$ & 0.752 & 0.2461 & 0.734 & 0.2648 & 0.770 & 0.2278 \\
\hline $10^{-2}$ & 0.682 & 0.1904 & 0.686 & 0.2320 & 0.692 & 0.1824 \\
\hline 0.1 & 0.444 & 0.0702 & 0.447 & 0.0953 & 0.433 & 0.0707 \\
\hline 1 & 0.212 & 0.0150 & 0.201 & 0.0167 & 0.184 & 0.0165 \\
\hline$+\infty$ & 0.291 & 0.1953 & 0.286 & 0.1919 & 0.263 & 0.2053 \\
\hline
\end{tabular}

Table 5

Transmittances, $T r$, and Reflectances, $R$, of fibrous medium with $85 \%$ of porosity subjected to a diffuse incident radiative flux with $L_{\text {slab }} / d=8$.

\begin{tabular}{|c|c|c|c|c|c|c|}
\hline \multirow[t]{2}{*}{ Absorption parameter $x k_{2}$} & \multicolumn{2}{|c|}{ Single-RTE approach } & \multicolumn{2}{|c|}{ Multi-RTE approach } & \multicolumn{2}{|c|}{ Reference DSMC } \\
\hline & $T r$ & $R$ & $\operatorname{Tr}$ & $R$ & $\operatorname{Tr}$ & $R$ \\
\hline $10^{-4}$ & 0.712 & 0.2859 & 0.705 & 0.2934 & 0.705 & 0.2926 \\
\hline $10^{-2}$ & 0.638 & 0.2217 & 0.657 & 0.2474 & 0.626 & 0.2411 \\
\hline 0.1 & 0.409 & 0.0826 & 0.385 & 0.1028 & 0.374 & 0.0904 \\
\hline 1 & 0.175 & 0.0293 & 0.130 & 0.0284 & 0.124 & 0.0288 \\
\hline$+\infty$ & 0.223 & 0.3056 & 0.190 & 0.2736 & 0.169 & 0.2672 \\
\hline
\end{tabular}

95\% porosities has thickness $\left(L_{\text {slab }}=25 d\right)$ larger than that of $79 \%$ porosity sample $\left(L_{\text {slab }}=5.833 d\right)$ and thus they feature largest computation times. For weakly absorbing fibers $\left(x k_{2}<0.2\right.$ in Fig. 9), the single-RTE approach is faster than the multi-RTE approach. Inversely, for highly absorbing fibers $\left(x k_{2}>0.2\right.$ in Fig. 9), the multiRTE approach is computationally efficient. Only effective properties and radiative transfer calculations in the fluid phase (air) are relevant while those in the absorbing solid phase are insignificant. The most time consuming calculation in both approaches (singleand multi-RTE) concerns the calculation of the effective properties rather than the solution of the RTEs. The difference is orders of magnitudes [14]. However, the effective properties have to be de- 


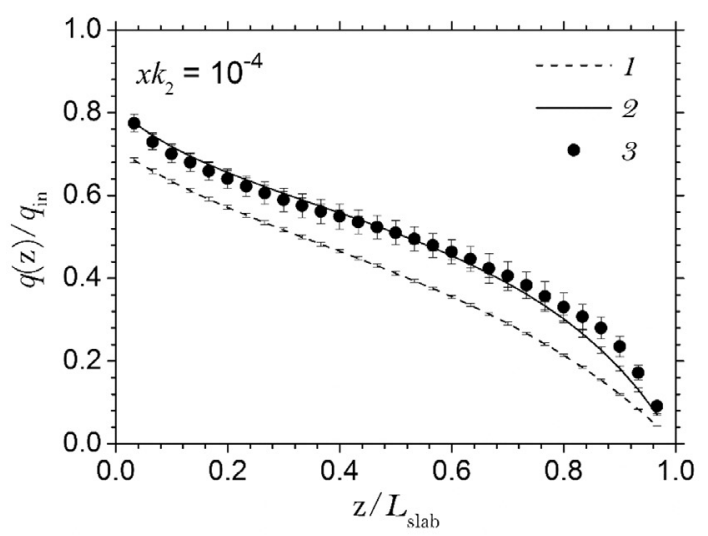

(a)

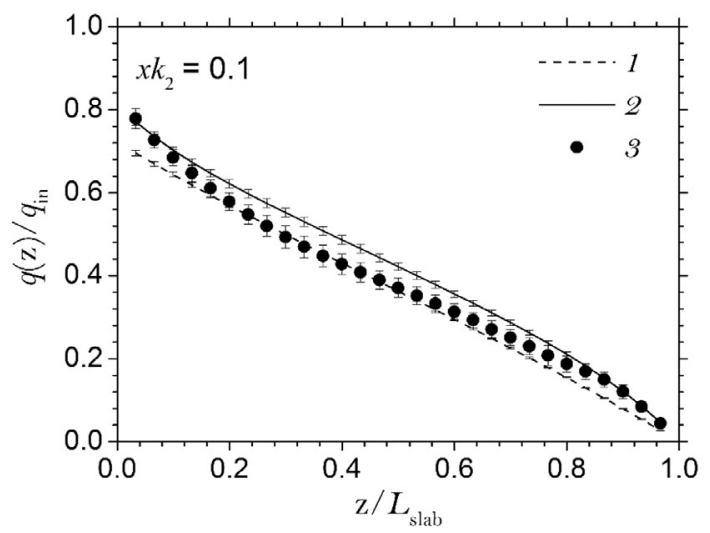

(b)

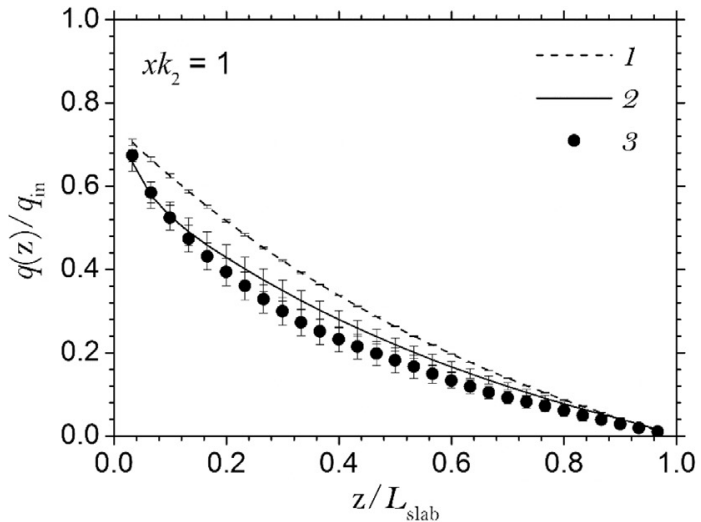

(c)

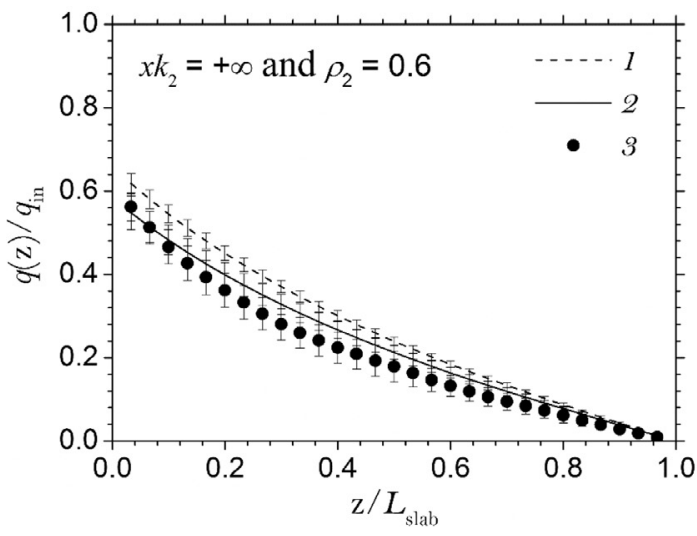

(d)

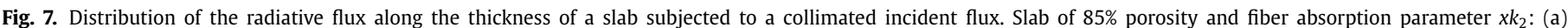

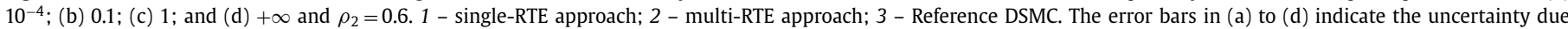

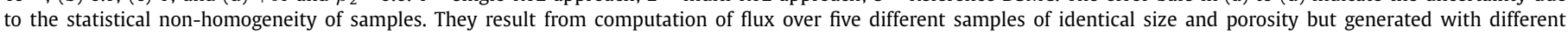
random seeds.

Table 6

Transmittances, $T r$, and Reflectances, $R$, of fibrous medium with $79 \%$ of porosity subjected to a diffuse incident radiative flux with $L_{\text {slab }} / d=5.833$.

\begin{tabular}{|c|c|c|c|c|c|c|}
\hline \multirow{2}{*}{ Absorption parameter $x k_{2}$} & \multicolumn{2}{|c|}{ Single-RTE approach } & \multicolumn{2}{|c|}{ Multi-RTE approach } & \multicolumn{2}{|c|}{ Reference DSMC } \\
\hline & $T r$ & $R$ & $T r$ & $R$ & $T r$ & $R$ \\
\hline $10^{-4}$ & 0.633 & 0.3647 & 0.629 & 0.3693 & 0.622 & 0.3757 \\
\hline $10^{-2}$ & 0.548 & 0.2856 & 0.561 & 0.3195 & 0.516 & 0.3167 \\
\hline 0.1 & 0.307 & 0.1198 & 0.262 & 0.1425 & 0.247 & 0.1397 \\
\hline 1 & 0.104 & 0.0605 & 0.063 & 0.0410 & 0.054 & 0.0499 \\
\hline$+\infty$ & 0.118 & 0.3626 & 0.098 & 0.3342 & 0.079 & 0.3138 \\
\hline
\end{tabular}

termined only once. The direct calculations (DSMC) take a similar amount of time and memory as the computation of the effective properties.

An analysis of the influence of the ray number used in the various Monte Carlo simulations on the standard deviation of continuum-scale and macroscopic radiative properties shown that $N=5 \times 10^{6}$ ray samples allow a numerical error due to finite ray number less than $1 \%$. The uncertainty due to the statistical nonhomogeneity of isotropic fibrous samples is investigated by carrying out computation over five different samples of identical size and porosity but generated with different random seeds. The results from these five different samples are then averaged out and the corresponding standard deviations are shown by error bars on Figs. 3, 5-8. It can be observed that the statistical nonhomogeneity of samples leads to important uncertainties on radiative flux depending on the depth and the absorption index of the fibers $k_{2}$. Typically, the largest standard deviations of the DSMC, the single and the multi-RTE approaches are about 25\%, 10\% and $15 \%$, respectively. It is worth noticing that the smaller the heat flux, the larger the standard deviation is. Consequently, large uncertainties associated to small radiative flux may not be meaningful on heat transfer calculations.

\section{Conclusion}

Radiative transfer in isotropic fibrous media is analyzed using continuum scale radiative properties determined either by volume averaging theories (refereed herein to as the multi-RTE approach) or by single-RTE approach. Fiber diameters are considered to be much larger than radiation wavelength so as for geometrical optics to be valid. The suitability of each approach is investigated by comparing the macroscopic optical properties, namely transmittances, 


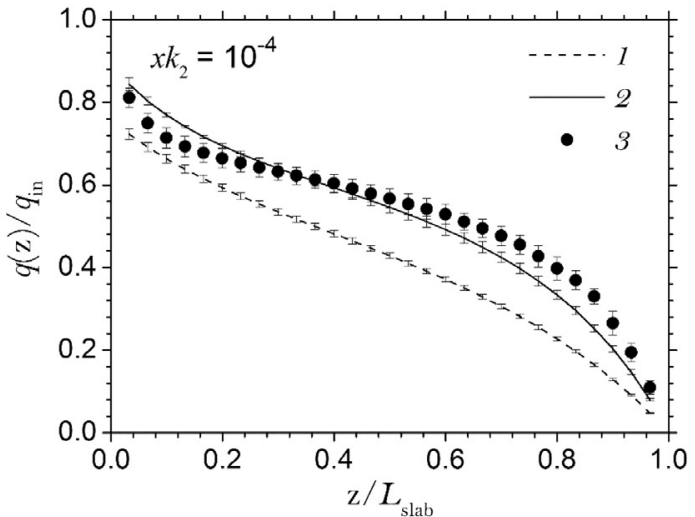

(a)

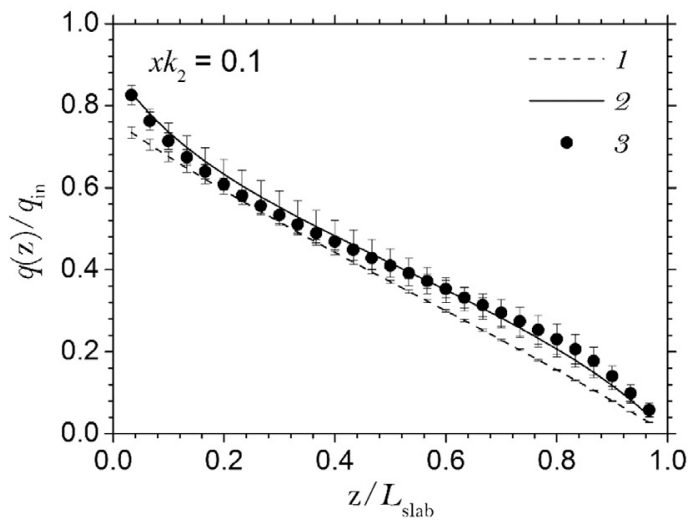

(b)

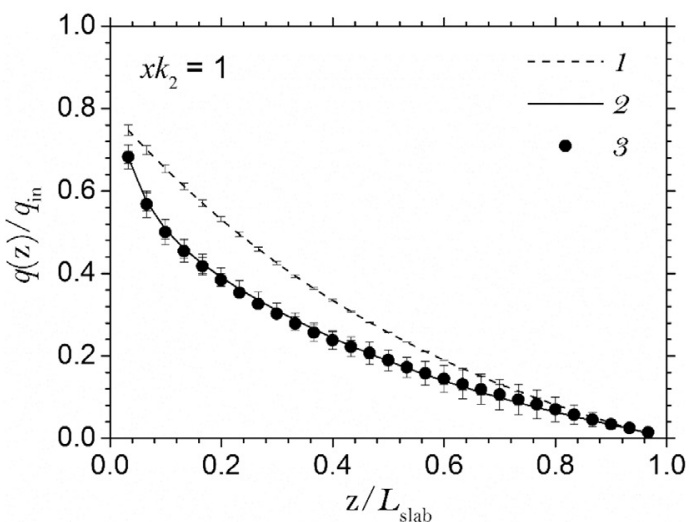

(c)

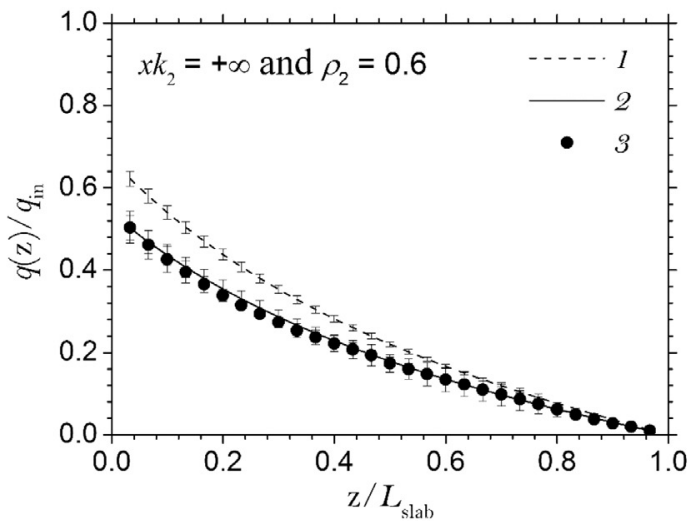

(d)

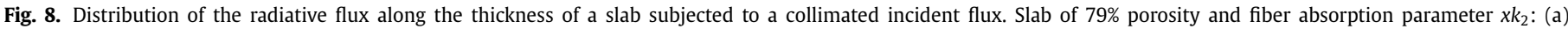

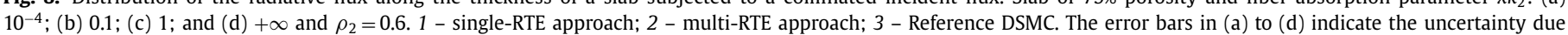

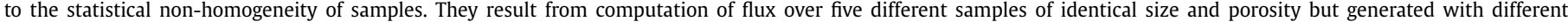
random seeds.

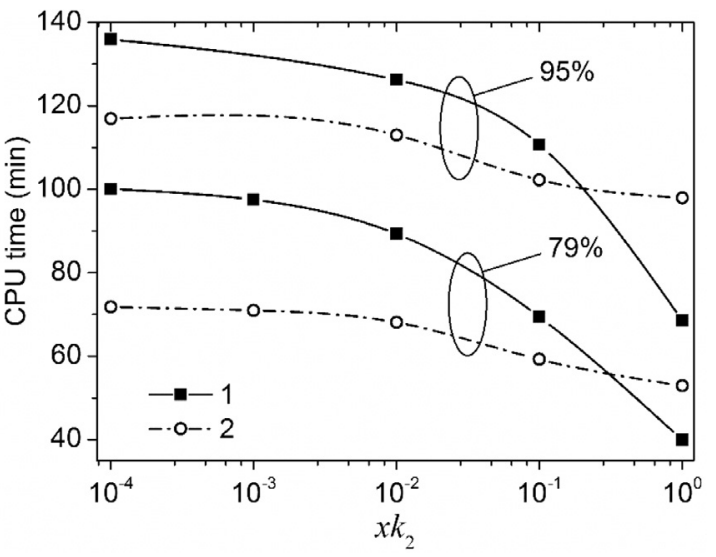

Fig. 9. Computation time per CPU versus the fiber absorption parameter $x k_{2}$ of (1) single- and (2) multi-RTE approaches for $95 \%$ and $79 \%$ porosity samples.

reflectance and radiative flux distributions. The direct simulation Monte Carlo of radiative transfer in 3D discrete fibrous slabs provides reference results. We conclude that:

The single-RTE approach is suitable for studying highly porous isotropic fibrous media (porosity equal to $95 \%$ here). The meanfree-path method enables to calculate the radiative properties of the equivalent homogeneous medium given the complex material features. As one set of effective radiative properties of a single phase is calculated only, straight forward application of the single-RTE approach is only given for cases where radiative transfer within the particle substance (e.g. fibers here) is not too significant.

- The multi-RTE approach is suitable for isotropic fibrous media of porosity in the range $79-95 \%$ and for realistic fiber optical properties. In addition, a set of effective radiative properties for each phase is used in this approach consequently, both radiative transfer in the fluid (air here) and the particle (fiber here) phases, and radiative exchange between them can be assessed using this multi-RTE approach.

- This study has revealed that the effective radiative properties in the solid phase of fibrous media in the geometric limit strongly depend on distribution of ray directions in this phase. The emitting medium scheme is suggested for determining effective properties of emitting fibrous media or fibrous media with irregular phase boundaries. In weakly emitting or cold fibrous media with optically smooth phase boundaries, the proposed cold medium scheme is recommended to determine appropriate effective properties.

\section{Acknowledgments}

The provision of high-performance computational resources, the Romeo HPC facility by the University of Reims ChampagneArdenne, and HPC Aries facility by the Ecole Polytechnique Fédérale de Lausanne (EPFL), is gratefully acknowledged. 


\section{Appendix A}

\section{A.1. Implementation of the inlet and outlet boundary effects in the path length Monte Carlo method}

One of geometric features of the REV is the fraction of solid phase on the boundaries, namely $f_{\mathrm{s}}$, which can be interpreted as the probability of a ray to reach a solid phase on the boundary. In path-length Monte Carlo method, an incident ray incoming on the inlet boundary strikes the solid phase boundary if the condition $f_{\mathrm{s}}>\zeta_{\mathrm{s}}$ is satisfied where $\zeta_{\mathrm{s}}$ is a random number drawn uniformly between 0 and 1 otherwise the ray enters the fluid phase and algorithm continues to follow the ray path.

When the ray hits the solid phase boundary, the algorithm calculate the reflectivity at the collision point, namely $\rho$, through the famous Fresnel formula [1,2] and compare it with a new random number $\zeta_{\rho}$. If $\rho>\zeta_{\rho}$ the ray is reflected by the current phase boundary else it is transmitted onto the adjacent phase. After the test, the algorithm continues to follow the ray path. Note that this local reflectivity is function of angle between the unit normal onto the boundary and the direction of the ray, and the refractive index of the fiber substance $n_{1}$ and $k_{1}$ given that the host medium has real refractive index of 1 . The same test applies also for any ray coming from the medium to the (inlet or outlet) boundary of the medium.

\section{References}

[1] Modest M. Radiative heat transfer. 3rd ed. New York: Academic Press; 2013.

[2] Howell J, Mengüç M, Siegel R. Thermal radiation heat transfer. 5th ed. New York: CRC Press; 2015.

[3] Lipiński W, Davidson JH, Haussener S, Klausner JF, Mehdizadeh AM, Petrasch J, et al. Review of heat transfer research for solar thermochemical applications. J Therm Sci Eng Appl 2013;5:021005

[4] Fend T, Hoffschmidt B, Pitz-Paal R, Reutter O, Rietbrock P. Porous materials as open volumetric solar receivers: experimental determination of thermophysical and heat transfer properties. Energy 2004;29:823-33.

[5] Furler P, Scheffe J, Gorbar M, Moes M, Vogt U, Steinfeld A. Solar thermochemical CO2 splitting utilizing a reticulated porous ceria redox system. Energy Fuels 2012;26:7051-9.

[6] Chahlafi M, Bellet F, Fichot F, Taine J. Radiative transfer within non Beerian porous media with semitransparent and opaque phases in non equilibrium: application to reflooding of a nuclear reactor. Int J Heat Mass Transfer 2012:55:3666-76.

[7] Haussener S, Gergely M, Schneebeli M, Steinfeld A. Determination of the macroscopic optical properties of snow based on exact morphology and direct pore-level heat transfer modeling. J Geophys Res 2012;117:F03009.

[8] Dombrovsky L, Randrianalisoa J, Lipiński W, Timchenko V. Simplified approaches to radiative transfer simulations in laser-induced hyperthermia of superficial tumors. Comput Therm Sci 2013;5:521-30.

[9] Viskanta R, Mengüç P. Radiative transfer in dispersed media. Appl Mech Rev 1989;42:241-59.

[10] Baillis D, Sacadura J-F. Thermal radiation properties of dispersed media: theoretical prediction and experimental characterization. J Quant Spectr Radiat Transfer 2000;67:327-63.

[11] Baillis D, Coquard R, Randrianalisoa J, Dombrovsky L, Viskanta R. Thermal radiation properties of highly porous cellular foams. Spec Top Rev Porous Media 2013;4:111-36.

[12] Consalvi JL, Porterie B, Loraud JC. A formal averaging procedure for radiation heat transfer in particulate media. Int J Heat Mass Transfer 2002;45:2755-68.

[13] Lipiński W, Petrasch J, Haussener S. Application of the spatial averaging theorem to radiative heat transfer in two-phase media. J Quant Spectr Radiat Transfer 2009;111:253-8.

[14] Petrasch J, Haussener S, Lipiński W. Discrete vs continuum level simulations of radiative transfer in semitransparent two-phase media. J Quant Spectr Radiat Transfer 2011;112:1450-9.

[15] Gusarov A. Homogenization of radiation transfer in two-phase media with irregular phase boundaries. Phys Rev B 2008;77 paper 144201.

[16] Randrianalisoa J, Baillis D. Radiative transfer in dispersed media: comparison between homogeneous phase and multiphase approaches. J Heat Transfer 2010;132 paper 023405.

[17] Lipiński W, Keene D, Haussener S, Petrasch J. Continuum radiative heat transfer modeling in media consisting of optically distinct components in the limit of geometrical optics. J Quant Spectrosc Radiat Transfer 2010;111:2474-80.
[18] Coquard R, Baillis D, Randrianalisoa J. Homogeneous phase and multiphase approaches for modeling radiative transfer in foams. Int J Therm Sci 2011;50:1648-63.

[19] Tong T, Tien C. Radiative heat transfer in fibrous insulations - Part I: analytical study. J Heat Transfer 1983;105:70-5

[20] Tong T, Yang Q, Tien C. Radiative heat transfer in fibrous insulations - Part II: experimental study. J Heat Transfer 1983;105:76-81.

[21] Lee S-Ch, Cunnington G. Conduction and radiation heat transfer in high-porosity fiber thermal insulation. J Thermophys Heat Transfer 2000;14:121-36.

[22] Lee S. Radiative transfer through a fibrous medium: allowance for fiber orientation. J Quant Spectrosc Radiat Transfer 1986;36:253-63.

[23] Boulet P, Jeandel G, Morlot G. Model of radiative transfer in fibrous media-matrix method. Int J Heat Mass Transfer 1993;36:4287-97.

[24] Milandri A, Asllanaj F, Jeandel G. Determination of radiative properties of fibrous media by an inverse method - comparison with the Mie theory. J Quant Spectrosc Radiat Transfer 2002;74:637-53.

[25] Tagne H, Baillis D. Radiative heat transfer using isotropic scaling approximation: application to fibrous medium. J Heat Transfer 2005;127:1115-23.

[26] Golombok M, Prothero A, Shirvill LC, Small LM. Surface combustion in metal fibre burners. Combust Sci Technol 1991;77:203-23.

[27] Suter S, Haussener S. Morphology engineering of porous media for enhanced solar fuel and power production. J Min Met Mat Soc 2013;65:1702-9.

[28] Mansour NN, Panerai F, Martin A, Parkinson DY, MacDowell A, Fast T, et al. A new approach to light-weight ablators analysis: from micro-tomography measurements to statistical analysis and modeling, 44th AIAA thermophysics conference, fluid dynamics and co-located conferences, (AIAA 2013-2768).

[29] Nouri N, Martin A. Three dimensional radiative heat transfer model for the evaluation of the anisotropic effective conductivity of fibrous materials. Int J Heat Mass Transfer 2015;83:629-35.

[30] Coquard R, Baillis D. Radiative properties of dense fibrous medium containing fibers in the geometric limit. J Heat Transfer 2006;128:1022-30.

[31] Arambakama R, Tafreshia H, Pourdeyhimi B. Analytical Monte Carlo ray tracing simulation of radiative heat transfer through bimodal fibrous insulations with translucent fibers. Int J Heat Mass Transfer 2012;55:7234-46.

[32] Nisipeanu E, Jones P. Monte Carlo simulation of radiative heat transfer in coarse fibrous media. J Heat Transfer 2003;125:748-52.

[33] Dauvois Y, Rochais D, Enguehard F, Taine J. Statistical radiative modeling of a porous medium with semi transparent and transparent phases: application to a felt of overlapping fibres. Int J Heat Mass Transfer 2016;106:601-18.

[34] Tancrez M, Taine J. Direct identification of absorption and scattering coefficients and phase function of a porous medium by a Monte Carlo technique. Int J Heat Mass Transfer 2004;47:373-83.

[35] Zeghondy B, Iacona E, Taine J. Determination of the anisotropic radiative properties of a porous material by radiative distribution function identification (RDFI). Int J Heat Mass Transfer 2006:49:2810-19.

[36] Haussener S, Lipiński W, Petrasch J, Wyss P, Steinfeld A. Tomographic characterization of a semitransparent-particle packed bed and determination of its thermal radiative properties. J. Heat Transfer 2009;131:072701.

[37] Berg MJ, Sorensen CM, Chakrabarti A. A new explanation of the extinction paradox. J Quant Spectrosc Radiat Transfer 2011;112:1170-81.

[38] Hottel H, Sarofim A. Radiative transfer. New York: McGraw-Hill; 1967.

[39] Quintard M, Whitaker S. Transport in ordered and disordered porous media II: generalized volume averaging. Transp Porous Media 1994

[40] Randrianalisoa J, Baillis D. Radiative properties of densely packed spheres in semitransparent media: a new geometric optics approach. J Quant Spectr Radiat Transfer 2010;111:1372-88.

[41] Banerji N, Leyland P, Haussener S. Tomography based radiative characterisation of decomposing carbonaceous heat shield materials. Carbon 2017;122:451-61.

[42] Van de Hulst H. Light scattering by small particles. New York: Dover; 1981.

[43] Kerker M. The scattering of light and other electromagnetic radiation. New York: Academic Press; 1969.

[44] Zarrouati M, Enguehard F, Taine J. Statistical characterization of near-wall radiative properties of a statistically non-homogeneous and anisotropic porous medium. Int J Heat Mass Transfer 2013;67:776-83.

[45] Sudiarta IW, Chylek P. Mie-scattering efficiency of a large spherical particle embedded in an absorbing medium. J Quant Spectr Radiat Transfer 2001;70:709-14

[46] Lebedev N, Gartz M, Kreibig U, Stenzel O. Optical extinction by spherical particles in an absorbing medium: application to composite absorbing films. Eur Phys J D 1999;6:365-73.

[47] Randrianalisoa J, Baillis D, Pilon L. Modeling radiation characteristics of semitransparent media containing bubbles or particles. J Opt Soc Am A 2006:23:1645-56.

[48] Farmer J, Howell J. Comparison of Monte Carlo strategies for radiative transfer in participating media. Adv Heat Transfer 1998;31:333-429.

[49] Brewster Q. Volume scattering of radiation in packed beds of large, opaque spheres. J Heat Transfer 2004;126:1048-50.

[50] Randrianalisoa J, Baillis D. Analytical model of radiative properties of packed beds and dispersed media. Int J Heat Mass Transf. 2014;70:264-75.

[51] Romeo HPC Center - Champagne-Ardenne.

[52] Aries cluster - Ecole Polytechnique Fédérale de Lausanne. 